\title{
Une grille chorématique des dynamiques spatiales pour expliquer l'évolution des territoires
}

A spatial dynamics chorematic grid to explain territories evolution

Un conjunto de coremas de dinámica espacial para explicar la evolución territorial

\section{Laure Casanova Enault et Cathy Chatel}

\section{OpenEdition Journals}

Édition électronique

URL : http://journals.openedition.org/mappemonde/2189

DOI : 10.4000/mappemonde.2189

ISSN : 1769-7298

\section{Éditeur}

UMR ESPACE

\section{Référence électronique}

Laure Casanova Enault et Cathy Chatel, « Une grille chorématique des dynamiques spatiales pour expliquer l'évolution des territoires », Mappemonde [En ligne], 119 | 2017, mis en ligne le 01 janvier 2017, consulté le 02 juillet 2020. URL : http://journals.openedition.org/mappemonde/2189; DOI : https://doi.org/10.4000/mappemonde.2189

Ce document a été généré automatiquement le 2 juillet 2020

\section{cc) (†)}

La revue Mappemonde est mise à disposition selon les termes de la Licence Creative Commons Attribution - Pas d'Utilisation Commerciale - Partage dans les Mêmes Conditions 4.0 International. 


\title{
Une grille chorématique des dynamiques spatiales pour expliquer l'évolution des territoires
}

\author{
A spatial dynamics chorematic grid to explain territories evolution \\ Un conjunto de coremas de dinámica espacial para explicar la evolución \\ territorial
}

Laure Casanova Enault et Cathy Chatel

\section{NOTE DE L'AUTEUR}

Les scénarios de prospective ayant été réalisés en 2012 et la modélisation graphique en 2013, les évolutions territoriales postérieures à cette période ne sont pas prises en compte.

1 La modélisation graphique de scénarios prospectifs consiste à confronter des configurations territoriales données à des changements possibles (variation démographique, transformation des infrastructures, évolution différenciée d'un phénomène, évènement spatial... (EPEES, 2000)) en étudiant leurs efFets sur l'évolution du territoire. Il est attendu de l'exploration de futurs possibles à travers des scénarios contrastés ${ }^{1}$, qu'elle indique comment s'opère la transition entre une situation présente et une situation projetée dans le futur. Le but est de permettre aux acteurs, en particulier de l'aménagement du territoire, de repérer les processus à maîtriser afin d'orienter les territoires vers la trajectoire souhaitée. La modélisation prospective nécessite donc d'une part, de décomposer les dynamiques de changement en jeu et, d'autre part, d'imaginer les dirférentes traductions territoriales qu'elles peuvent admettre. Il peut s'agir de changements de forme ou de changements de localisation de phénomènes et d'objets (dont l'éventail des cas possibles est borné par les contraintes 
de l'espace), ainsi que de changements d'état, lesquels constituent un défi important de l'exploration prospective.

2 Afin d'appliquer cette approche aux scénarios du programme national de prospective Territoire Durable $2030^{2}$, nous avons fait le choix de produire une grille de chorèmes spatio-temporels. Établie par approche hypothético-déductive, cette grille décompose les éléments clés d'une dynamique spatiale de changement et montre que ces éléments peuvent varier selon deux modalités possibles au minimum, légitimant ainsi l'existence de plusieurs scénarios d'évolution d'un territoire.

\section{Méthode pour la modélisation des dynamiques spatiales des scénarios Territoire Durable 2030}

3 Le programme Territoire Durable 2030, piloté par la mission prospective du Ministère de l'Écologie, du Développement Durable et de l'Énergie (MEDDE), interroge les modalités d'un développement durable du territoire national à l'horizon 2030 (CGDD, 2013). Il s'est amorcé par une démarche de prospective experte ${ }^{3}$ conduite par un bureau d'étude dont le résultat est un rapport présentant sous forme narrative le contexte de développement de la France à l'horizon 2030 ainsi que 4 scénarios (BIPE, 2012). Ensuite, différents prestataires de services ont été chargés de l'analyse et de la cartographie d'indicateurs de durabilité. Dans cette phase de travail, les chercheurs de l'UMR Espace (UMR ESPACE et al., 2013) ont apporté un éclairage géoprospectif aux scénarios de prospective (notamment par la réalisation de simulations de la pression urbaine dans chacun des scénarios et par la modélisation graphique des scénarios).

4 Ainsi, à l'issue de la première phase de prospective experte, trois des scénarios étudiés ont été soumis à l'exercice de modélisation graphique. L'objectif est d'introduire les dimensions spatiale et systémique des changements envisagés afin d'étudier les modalités de territorialisation des scénarios narratifs (CGDD, 2012). La modélisation graphique est donc employée dans une finalité un peu différente de la composition de modèles d'organisation de l'espace: l'enjeu est de se focaliser sur l'éventail des dynamiques spatiales susceptibles de générer du changement dans l'évolution du territoire.

5 Cette modélisation comprend quatre rubriques correspondant aux quatre variables clés que nous avons identifiées dans les scénarios : les réseaux urbains, les axes et les flux, la gouvernance et l'administration territoriale, enfin la ressource et les pressions environnementales. Le travail s'opère en trois temps. D'abord, nous avons fait le choix de modéliser la situation de 2010 pour modéliser ensuite les situations projetées en 2030. Le troisième temps constitue l'exercice le plus original et approfondi du travail de modélisation réalisé : il fonde la grille chorématique. Pour chacune des rubriques, les modalités de transition entre les structures et les dynamiques en cours en 2010 et celles projetées en 2030 sont analysées. Cela revient par exemple à questionner les processus capables de modifier la dynamique d'urbanisation et de métropolisation en cours en 2010 pour tendre vers celle de "déconcentration", voire dans certains cas de « désurbanisation », imaginée en 2030 (scénario des territoires inversés), ou encore de modifier la logique de coopération entre certains pôles urbains à l'œuvre en 2010, pour tendre vers une valorisation des réseaux urbains au sein de leurs régions en 2030 (scénario des territoires singuliers). 
6 Pour être explicatif, le modèle prospectif ne peut se contenter de signaler une dynamique, il doit préciser les processus en jeu. Dans le premier cas, l'émergence de nouveaux pôles dans l'espace rural et, dans le second cas, le développement de relations horizontales entre les territoires comptent parmi les processus de transition possibles. Il est également nécessaire de pointer les changements territoriaux induits par les dynamiques identifiées afin de livrer aux décideurs les éléments leur permettant de juger si l'évolution territoriale considérée est souhaitable ou non.

7 Enfin, si de nombreux processus interviennent dans une dynamique, une difficulté se pose pour les représenter sur un modèle statique, d'autant plus lorsque plusieurs dynamiques sont en jeu. C'est bien là l'intérêt de leur décomposition dans le cheminement de modélisation graphique précédent la composition du modèle final. Dans cette dernière figure en revanche, seuls les principaux efFets du changement opéré sur le territoire seront représentés. Dans le cas d'une dynamique de déconcentration, le phénomène de perte d'attractivité d'un lieu au profit d'un autre entraîne un déplacement de populations selon une certaine direction, produisant une nouvelle géographie urbaine. Dans le scénario des territoires inversés, l'investissement par les populations d'espaces ruraux nouvellement attractifs constitue le changement le plus décisif, alors que dans le scénario des territoires singuliers, la concentration métropolitaine prime.

8 Aussi, le choix a été fait d'établir un référentiel de chorèmes des éléments qui interviennent dans une dynamique spatiale. Le but est de décomposer les processus qui entrent dans les dynamiques des scénarios dans une perspective nomothétique. Se pose en efFet la question de savoir si la chorématique peut utilement éclairer la prospective par la formalisation de structures spatio-temporelles élémentaires qui entrent dans l'explication de l'évolution des territoires. Ce questionnement prolonge les réflexions sur la représentation des dynamiques des territoires (Kaddouri et al., 2014) et celles amorcées au début des années 2000 sur la place des représentations graphiques en prospective (Bailly et al., 2001 ; Debarbieux et al., 2002 ; Hurel, 2010).

\section{Une grille de chorèmes spatio-temporels pour modéliser les dynamiques de scénarios prospectifs}

9 La table des chorèmes spatio-temporels a pour but d'établir un référentiel permettant de modéliser les dynamiques spatiales qui marquent une modification de la trajectoire d'évolution du territoire et plus largement, la manière dont leur association définit la structure spatio-temporelle dans laquelle s'inscrit cette évolution. Il s'agit là d'enrichir les référentiels existants (Brunet, 1986; Cheylan et al., 1990) tout en cherchant à répondre aux besoins de la modélisation prospective. Les contributions plus ou moins récentes pour une meilleure prise en compte du temps dans les modèles graphiques ne se sont en efFet pas révélées directement transférables à l'exercice prospectif mené. La chronochorématique (Théry, 1990; Rodier et al., 2010-2014) s'appuie sur l'étude des changements fonctionnels locaux qui déterminent l'évolution de villes pour rendre compte de l'héritage spatial de leur trajectoire d'évolution sur un unique modèle spatial. L'approche est particulièrement efFIcace pour l'étude d'organisations spatiales à grande échelle. D'autres travaux identifient des situations géo-historiques modèles (Grataloup, 1996), comme la situation d'isthme ou d'angle-mort, dont la récurrence au cours de l'histoire amène à définir de véritables trajectoires géo-historiques. Ces 
situations géo-historiques se distinguent des dynamiques de changement étudiées ici à la fois du point de vue de la temporalité de temps long considérée (Durand-Dastès, 1999) et du point de vue des items de la modélisation retenus lesquels sont liés à la finalité de la modélisation: intégrer des facteurs spatiaux dans l'explication de la trajectoire historique des États. Des propositions ont également été faites en matière de sémiologie graphique animée des flux (Kaddouri, 2008). Par animation graphique, l'auteur met en évidence l'intérêt, déjà formalisé par d'autres (Allen, 1983; Cheylan et al. 1997 ; Cassini, 1999 ; Peguy, 2001), de différencier la continuité et la discontinuité spatio-temporelle des processus. Cette approche est utile pour qualifier l'efFet possible des dynamiques sur la trajectoire des territoires. La grille chorématique appliquée à l'espace rural (Cheylan et al., 1990) présente l'intérêt de décomposer deux temporalités des dynamiques : celle des processus et celle de la succession des états représentant des changements. Les types de changements territoriaux induits restent à préciser pour que la grille constitue un référentiel suffisant pour la modélisation des scénarios prospectifs.

10 Au delà de la table créée par Brunet (1986), l'examen des travaux de Cheylan (2007) et de Maby (2002) nous a permis d'alimenter la réflexion préalable à la conception de la grille chorématique des dynamiques spatiales. Développant respectivement un travail sur l'introduction du temps dans les modèles géographiques et spatio-systémiques, ils proposent des représentations spatio-temporelles qui ont été reprises pour développer et systématiser les chorèmes relatifs aux dynamiques de changement dans la table que nous avons produite (les chorèmes hérités des travaux de ces auteurs sont signalés en grisé sur la figure 1). Précisément :

- Quatre chorèmes de la table de Brunet (sur 28 au total) se rapportent à la dynamique spatiale et sont relatifs à l'expansion d'un phénomène dans l'espace (cf. dimension 3 de la figure 1) et à la qualité des évolutions localisées (cf. dimension 1 de la figure 1).

- Les trois temporalités des territoires mises en évidence dans les travaux de Cheylan (2007) permettent de difFérencier trois temps des dynamiques spatiales : celles qui se rapportent à la « vie » (cf. dimensions 1 et 2 de la figure 1), à la « trajectoire » (cf. dimensions 3 et 4 de la figure 1) et à la " généalogie » (cf. dimension 5 de la figure 1) des objets géographiques.

- Les « processeurs spatio-systémiques » développés par Maby, dont la figuration est inspirée du langage chorématique, alimentent la réflexion sur les concepts clés du changement spatial (2002, p. 147). L'auteur établit une grille de processeurs pour expliquer les dynamiques du foncier rural, viticole en particulier. Une rubrique se rapporte aux «relations spatio-systémiques» (dont l'esprit correspond à la dimension 4 citée) correspondant aux processus d'échange et aux relations logiques, tandis qu'une autre décrit le «changement spatio-systémique » : il y difFérencie les processus de positionnement, de mouvement et de différenciation spatiale (que l'on retrouve dans les dimensions 3 et 5 de la figure 1).

11 La table des chorèmes spatio-temporels décline ainsi cinq dimensions des dynamiques (en ligne) pour trois formes spatiales fondamentales ${ }^{4}$ (en colonne - le point, la ligne, l'aire) définies dans la table des chorèmes originelle de Brunet. Chacune des dimensions est déclinée de façon binaire (évolution "positive »/évolution "négative ») dans une logique de simplification des tendances possibles. Cette représentation laisse néanmoins toute la place à la déclinaison d'états intermédiaires, qui constituent autant de trajectoires possibles pour des scénarios de prospective. 
Figure 1. Grille de chorèmes spatio-temporels pour la modélisation graphique des dynamiques spatiales du changement

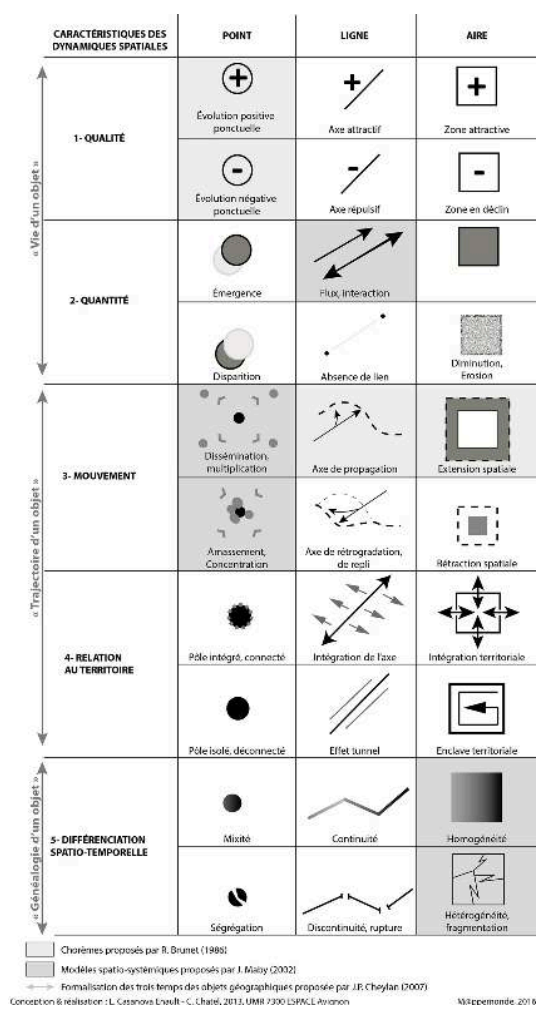

Conception et réalisation : L. Casanova Enault - C. Chatel, 2013, UMR 7300 ESPACE Avignon

Les dimensions 1 et 2 affectent la qualité (évolutions positive ou négative) et la quantité (intensité du phénomène généré) des dynamiques. Elles renseignent sur le contenu des objets, leur "vie " (cf. terminologie de Cheylan, également utilisée par Bonin en 2001). La dimension 3 se rapporte au mouvement représentant les modifications matérielles des objets spatiaux, principale dimension prise en compte dans la table de Brunet (1986) et développée dans la table de Cheylan et al. (1990). Les dimensions 4 et 5 décrivent la configuration spatiale résultante d'un processus. La dimension 4 intègre la dimension «spatio-systémique » relevée dans les travaux de Maby. Elle renvoie à la manière dont est internalisé le changement dans le fonctionnement du territoire et se réfère en particulier à une dimension immatérielle des dynamiques. Enfin, la dimension 5 se réfère à la "généalogie " des objets en figurant les phénomènes de différenciation spatiale réalisés au cours du temps.

Cette grille chorématique permet ainsi de répondre au besoin, en prospective comme dans toute analyse spatio-temporelle, de connaître les caractéristiques des dynamiques de changement à travers leur qualité et leur quantité, les mouvements ou changements d'état induits et la configuration spatiale résultante sachant que seul le changement ayant un impact majeur sur le territoire pourra être représenté. 


\section{Les chorèmes spatio-temporels éprouvés dans la modélisation graphique des scénarios prospectifs Territoire durable 2030}

Un exemple de l'application de cette grille chorématique (figure 1) à la donne urbaine du territoire est extrait ${ }^{5}$ de la modélisation graphique des scénarios Territoire Durable 2030. Cet exemple doit permettre d'évaluer l'utilité de la grille chorématique pour modéliser les dynamiques à l'origine des changements entre la situation de 2010 et les scénarios projetés en 2030. Le contexte de modélisation prospective influence l'approche hypothético-déductive habituellement adoptée en modélisation graphique. L'aller-retour s'opère toujours entre les observations de la réalité et le modèle du territoire en 2010. La spécificité est que ce dernier est aussi pris comme référence pour la modélisation des scénarios de 2030. La structure et les dynamiques du territoire national en 2010 sont à la fois objet de la modélisation et modèle de référence (sorte de chorotype adapté à l'exercice prospectif) pour la modélisation prospective. Cela implique que les rubriques de la modélisation de 2010 permettent la compréhension des dynamiques à 2030 .

15 Afin d'illustrer l'apport de la grille chorématique, la modélisation des scénarios n'est pas exposée dans son intégralité. L'exemple concerne la première rubrique des scénarios : celle se rapportant aux réseaux urbains (figure 2) du territoire.

Figure 2. Modélisation graphique des réseaux urbains en France en 2010

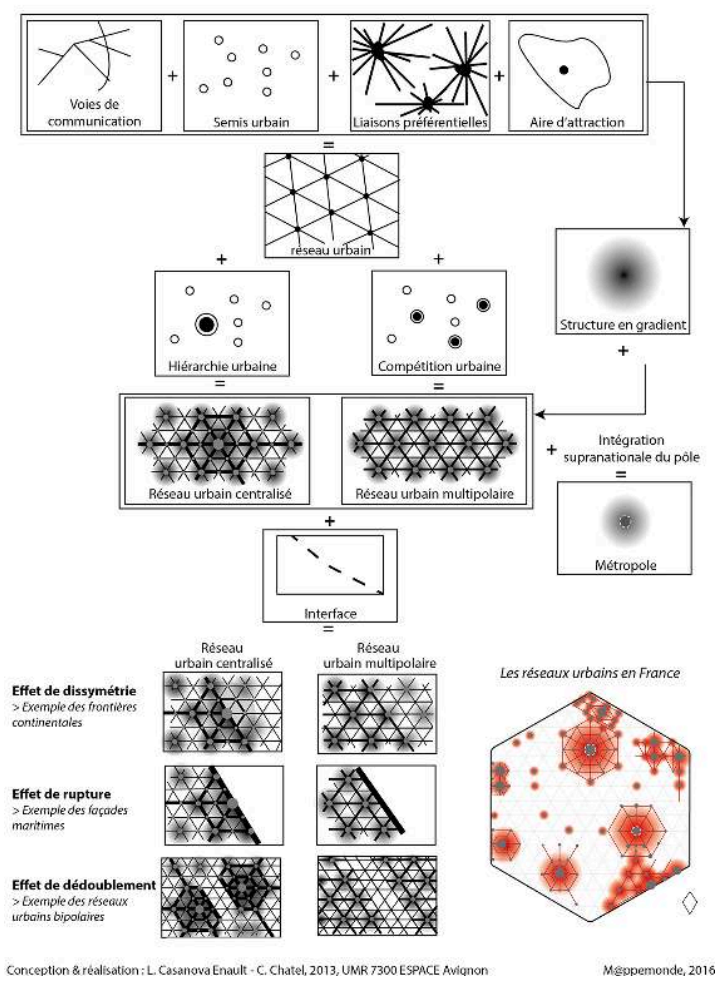

Conception et réalisation : L. Casanova Enault - C. Chatel, 2013, UMR 7300 ESPACE Avignon

Quatre chorèmes sont sélectionnés dans la table de référence de Brunet pour expliquer la logique de formation et de déploiement spatial des réseaux urbains formés à l'échelle 
métropolitaine. La présence d'un semis urbain doublé de celle de voies de communication traduit le principe d'implantation préférentielle des pôles urbains au croisement de voies de communication, soit un modèle de carrefour. Par extension, ce modèle donne lieu à une forme plus complexe, le réseau maillé. Associés aux chorèmes de liaisons préférentielles entre villes et d'aires d'attraction, il devient possible de comprendre la logique de formation des réseaux urbains. Celle-ci prend appui sur un treillage du territoire et donne lieu, sous l'effet de polarisation, à la formation de structures en gradient auréolaire signifiant le degré d'intensité des relations inversement proportionnelle à la distance au pôle urbain qui s'établit entre les centres et les périphéries urbaines.

La présence d'un seul ou de plusieurs pôles dominants dans le semis de villes différencie deux types de réseaux urbains : centralisé, lorsque la hiérarchie urbaine est marquée, et multipolaire, lorsque des relations mêlant complémentarité et compétition entre les pôles se traduit par un équilibre entre ceux-ci et par un réseau de villes de poids équivalents. L'intégration supra-nationale du ou des pôles principaux indique la présence d'une métropole. Dans ce cas, les réseaux, qu'ils soient centralisés ou multipolaires, sont dits métropolitains.

Lorsqu'ils sont replacés dans le contexte territorial français, les réseaux urbains s'ajustent plus ou moins fidèlement au modèle théorique. On dénombre trois principales déformations liées à l'efret de dissymétrie que l'on retrouve dans le cas des frontières continentales (réseaux urbains dans le prolongement de Strasbourg ou de Lille, en Allemagne et en Belgique), à l'efFet de rupture que l'on observe lorsque les réseaux urbains se déploient sur des façades maritimes (réseau urbain sud-est, déployé le long de la façade méditerranéenne) et enfin à l'erfet de dédoublement qui se manifeste dans le cas des réseaux urbains bipolaires (réseau urbain articulé autour de Rennes et Nantes).

19 Le modèle graphique des réseaux urbains en France établi pour 2010 est ensuite confronté au contexte urbain décrit dans les trois scénarios du programme Territoire Durable 2030 : territoires leviers - qui correspond au scénario tendanciel -, territoires singuliers, territoires inversés. Dans le scénario des «territoires leviers ", qui postule une reprise de la croissance, l'armature territoriale se restructure autour des métropoles et des infrastructures régionales, et les acteurs poursuivent leurs politiques d'innovation et d'attractivité internationale (pôles de compétitivité, etc.). La France de 2030 est constituée de territoires qui gagnent le pari de la mondialisation, et de territoires qui perdent. L'idée-force du scénario des « territoires singuliers » est que les territoires coopèrent de manière intelligente à travers un fonctionnement en «mode projet ». Les " territoires inversés ", décrits dans le troisième scénario, procèdent de la recherche d'un cadre de vie soutenable, au-delà des limites urbaines où les tensions sociales s'accentuent. On assiste à un exode urbain important et à une relocalisation à la campagne, permise par l'essor des NTIC, du travail à distance et des modes de consommation privilégiant les circuits courts.

Trois sources différentes alimentent la modélisation des scénarios: le déroulé des scénarios restitué sous forme narrative (rapport du BIPE, 2012 ; CGDD, 2012 et 2013), les projections de variables (démographiques, économiques, liées aux modes de vie etc.) réalisées à partir d'un modèle économétrique ${ }^{6}$ (BIPE, 2012) dont les résultats ont appuyé la réflexion pour l'écriture des scénarios et qui ont fait par ailleurs l'objet de cartographies thématiques ${ }^{7}$, les résultats cartographiques de simulations de la pression 
urbaine produites par la plateforme FRED $^{8}$ (Decoupigny, Passel, 2014). La modélisation graphique de la donne urbaine dans les trois scénarios de prospective consiste à mobiliser le minimum de chorèmes nécessaires pour comprendre les changements intervenus entre 2010 et 2030 conformément au principe de parcimonie propre à toute modélisation.

Dans le cas du scénario des territoires leviers, la qualité (dimension 1 de la grille des chorèmes) des dynamiques projetées en 2030 permet de comprendre le différentiel d'attractivité du territoire simulé. Ces tendances s'appuient sur les cartographies des projections démographiques et économiques réalisées dans le cadre du programme. La figure 3 illustre ainsi le passage des analyses réalisées en amont à la modélisation graphique des scénarios.

Figure 3. Exemples de schémas graphiques des projections économiques et démographiques à 2030 pour le scénario des territoires leviers

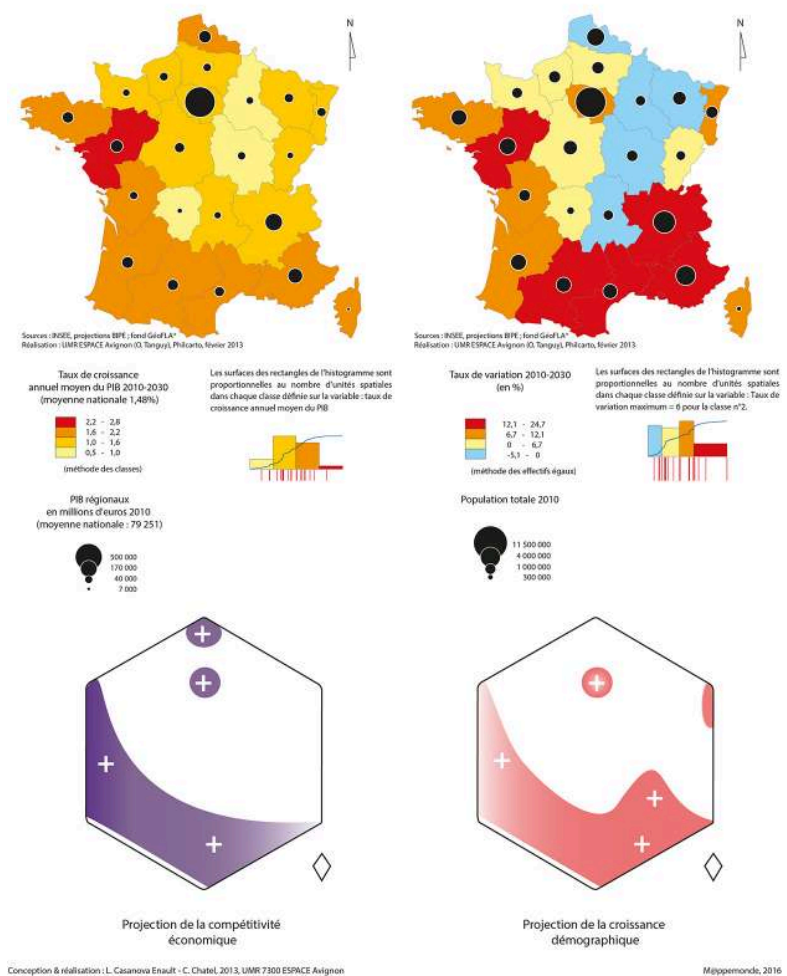

Sources : cartes (haut), réalisation : UMR ESPACE Avignon (O. Tanguy), Philcarto, février 2013; schémas (bas), conception et réalisation : L. Casanova Enault - C. Chatel, 2013, UMR 7300 ESPACE Avignon

Des schémas graphiques de ces cartes ont d'ailleurs pu être insérés dans la modélisation graphique (pour l'ensemble des quatre rubriques) afin de signifier, dès que cela a été possible, la rigueur de la démarche: chaque modèle intermédiaire produit résulte d'analyses des possibilités d'évolution du territoire. Le difFérentiel d'attractivité des territoires français est déterminant dans les changements qui afFectent les réseaux urbains dans le scénario levier (figure 4). Ainsi, les anciens socles industriels du Nord-Est et du centre, exclus de la croissance, sont en situation de repli, ce qu'illustre le modèle de l'enclave territoriale. La déconnexion entre ces espaces et les espaces aux marges du territoire français, intégrés à la dynamique internationale par le relais des métropoles régionales, constitue une seconde traduction des principaux 
changements permettant de saisir la transition entre la situation de 2010 et celle projetée en 2030 dans le scénario des territoires leviers.

Figure 4. Modélisation graphique de la donne urbaine dans le scénario des territoires leviers

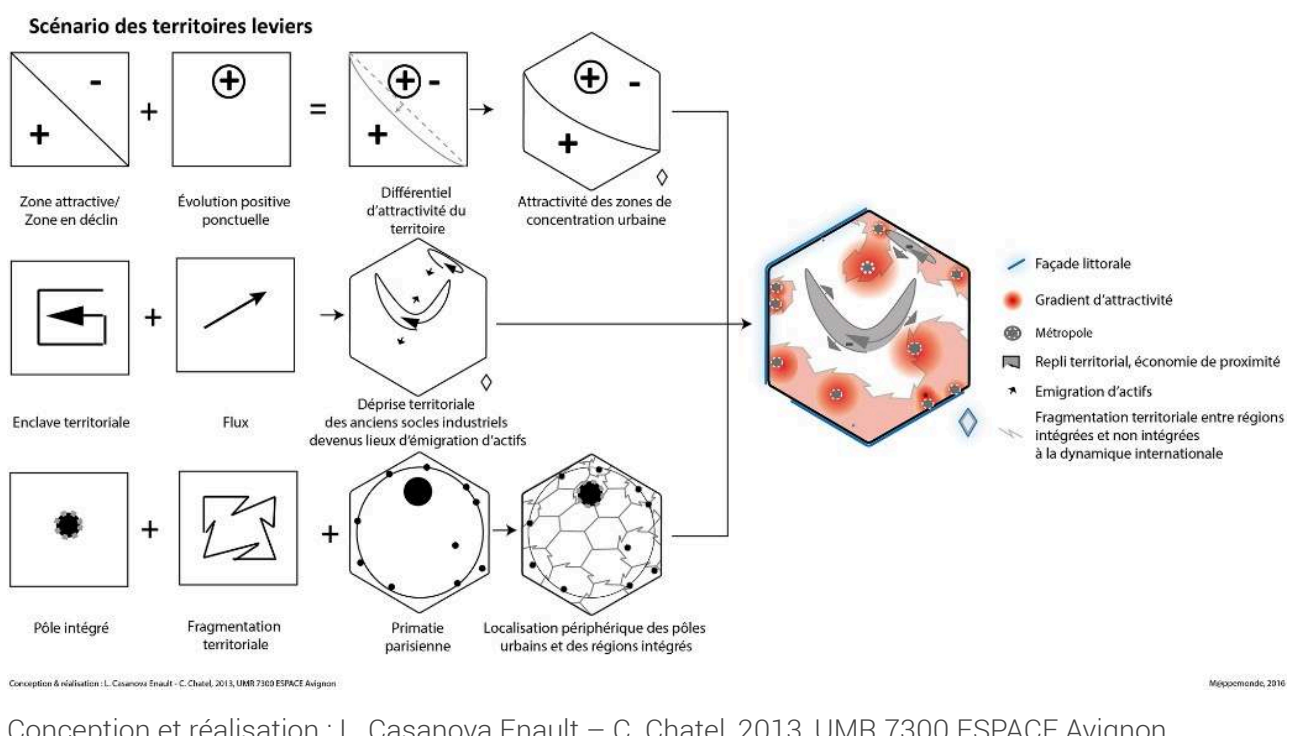

Dans le scénario des territoires singuliers, si la dynamique urbaine de 2030 (figure 5) se comprend là aussi à travers l'intégration des régions en marge de l'hexagone par les métropoles régionales, c'est l'intensification des échanges et le développement de relations de complémentarité au sein des régions, animées par leurs pôles, qui induit le plus fort changement. En efFet, le développement des relations horizontales (déclinaison du chorème de flux), facilité par le rôle de tête de pont des pôles régionaux, a permis le développement du mode de fonctionnement en réseau et, par voie de conséquence, la valorisation de nouvelles centralités dans l'espace rural. Cette dynamique marque le pas d'une trajectoire d'homogénéisation de la distribution des hommes et des activités dans le territoire suivant un scénario plus égalitaire. 
Figure 5. Modélisation graphique de la donne urbaine dans le scénario des territoires singuliers

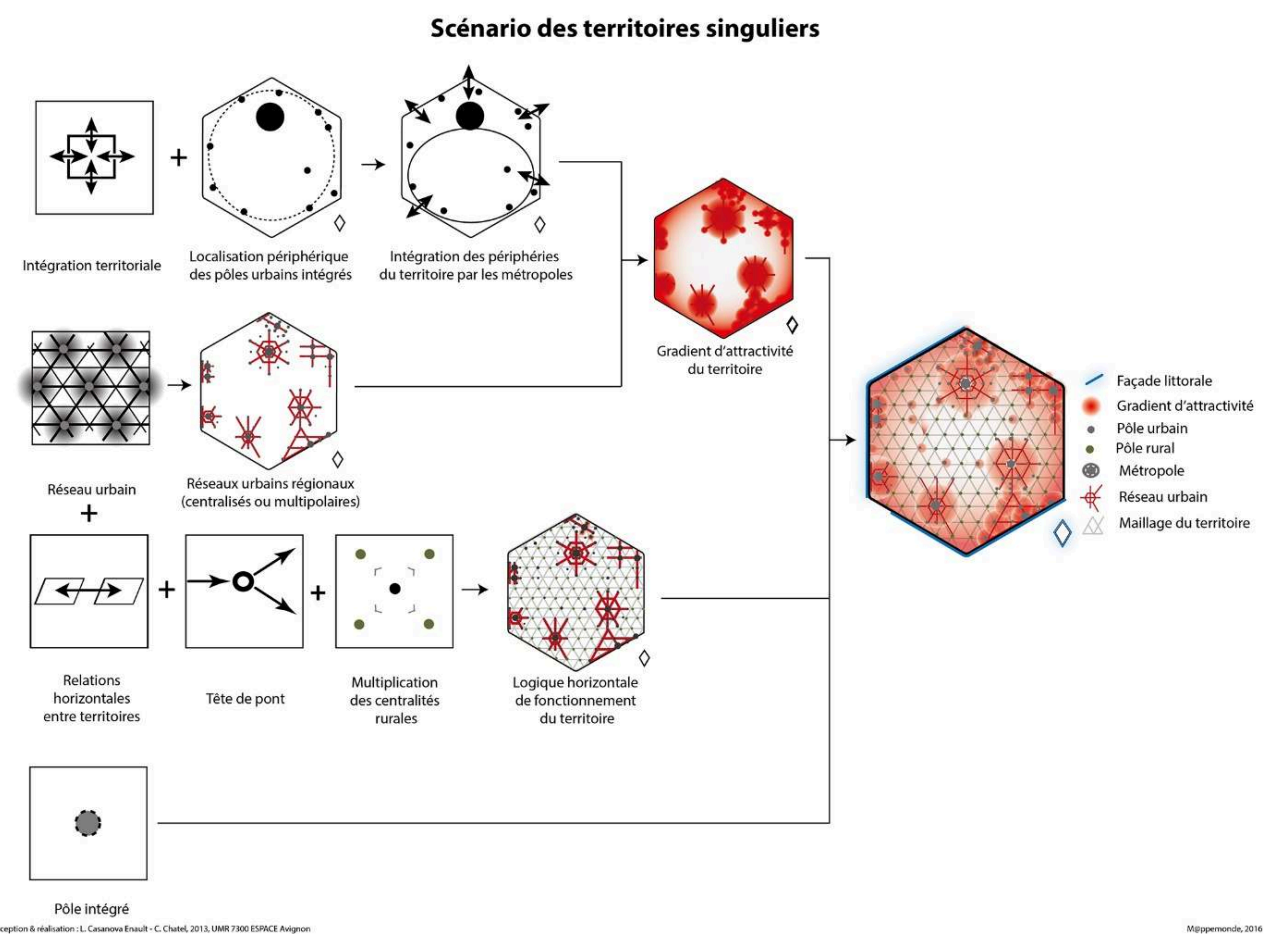

Conception et réalisation : L. Casanova Enault - C. Chatel, 2013, UMR 7300 ESPACE Avignon

Pour comprendre la transition vers le scénario des territoires inversés (figure 6), la plus radicale par rapport à la situation de 2010, il suffit de deux chorèmes décrivant l'efret d'un renversement de la logique d'attractivité du territoire : la répulsivité des centres urbains (due à plusieurs facteurs comme la saturation urbaine) et l'attractivité des espaces de faible densité. Un mouvement d'investissement de l'espace rural par les populations, doublé d'une déconcentration à partir des pôles urbains, produit une distribution plus équilibrée des hommes et des activités. 


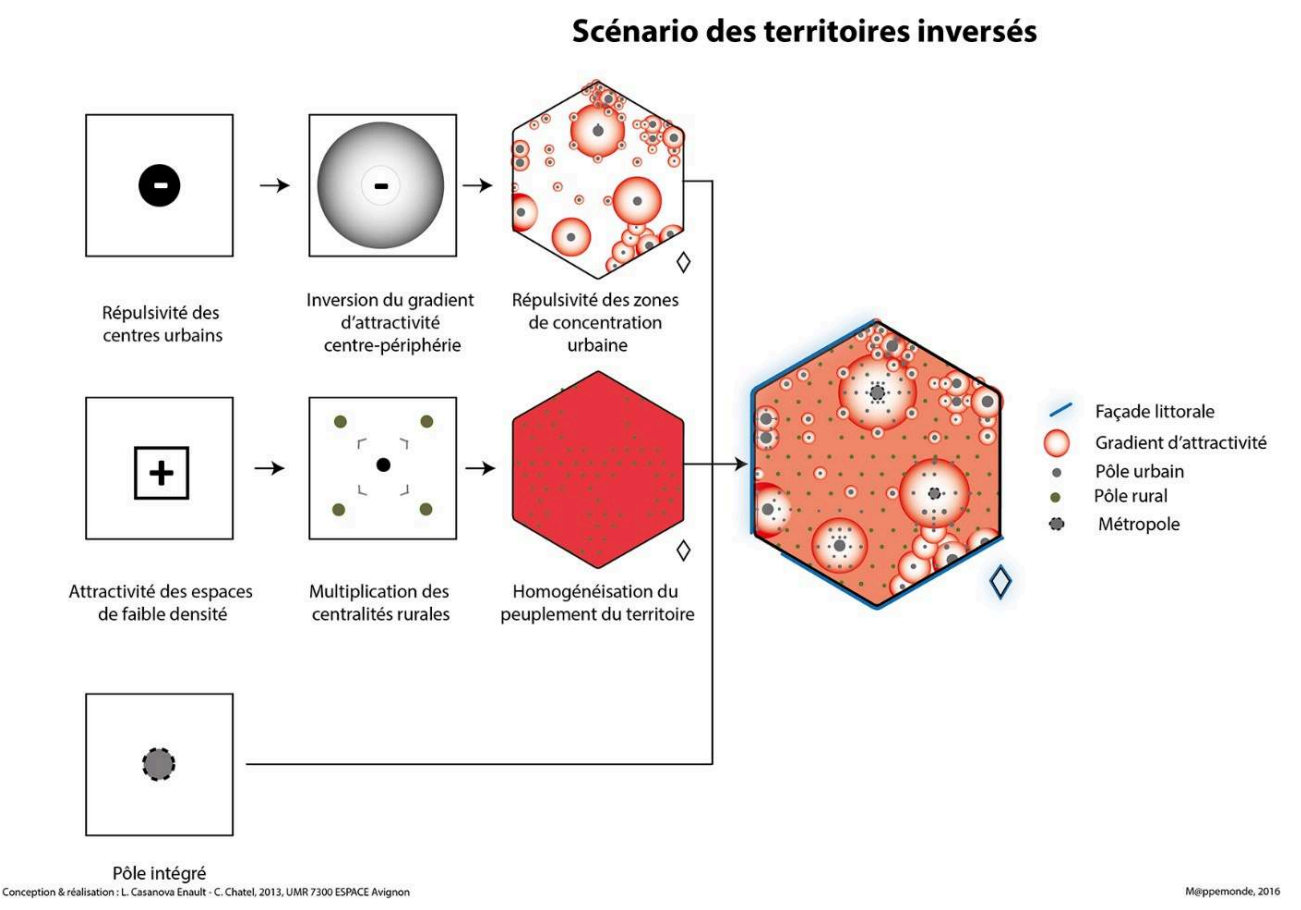

Conception et réalisation : L. Casanova Enault - C. Chatel, 2013, UMR 7300 ESPACE Avignon

Si les dynamiques en jeu dans les trois scénarios révèlent des processus similaires, ceux-ci n'aboutissent pas à la même image du territoire. Il importe en efFet de décomposer les différentes facettes des dynamiques spatiales pour comprendre les processus susceptibles de faire varier la trajectoire d'un territoire. Par exemple, si les scénarios des territoires singuliers et inversés dessinent une trajectoire d'homogénéisation de la distribution des hommes et des activités du territoire, et s'inscrivent donc dans la même structure spatio-temporelle, c'est sous l'erfet de processus différents. Les processus d'expansion du réseau de petites villes dans le scénario des territoires singuliers, et d'exode urbain ainsi que de répulsion des villes dans le scénario des territoires inversés, amènent à la même dynamique, soit une déconcentration et une distribution des hommes et des activités plus équilibrées. Ensuite, les efrets de cette dynamique se traduisent de manière différente. Dans le scénario des territoires singuliers, elle produit une multiplication des centres urbains, de relais ruraux avec une atténuation de la hiérarchie urbaine. Dans le scénario des territoires inversés, elle se traduit par un relatif exode urbain, l'investissement de la campagne par les métropolitains et un mode de vie local.

Autre exemple, les processus d'intégration des espaces en marge du territoire français à l'œuvre dans les scénarios des territoires leviers et singuliers ne produisent pas la même dynamique de changement, ni n'inscrivent les territoires dans la même trajectoire : on assiste à une évolution asymétrique des territoires pour l'un, et à un éclatement relatif des territoires lié à la valorisation du niveau micro local pour l'autre.

Si la méthode de modélisation graphique ne suffit pas en soi pour réaliser une exploration géoprospective, elle présente l'intérêt de territorialiser les scénarios, de localiser les espaces à enjeux, et enfin de formaliser les changements, y compris qualitatifs, qui déterminent les territoires du futur. D'autres investigations sur les 
dynamiques à l'œuvre à l'avenir doivent y être associées notamment afin de hiérarchiser, quantifier et spatialiser avec plus de précision leurs efFets.

La modélisation graphique des dynamiques spatiales enrichit l'approche de la modélisation prospective. Dans ce type d'exercice, plus que la représentation de l'image des territoires dans le futur, c'est une analyse compréhensive des dynamiques qui en sont à l'origine qui peut être proposée. Un jeu de dynamiques spatio-temporelles modèles permet de saisir les facteurs de changements ainsi que ses modalités. C'est l'objet de la grille chorématique élaborée : établir un référentiel de chorèmes pour révéler par la modélisation graphique comment une dynamique de changement peut produire des configurations spatiales différentes. Pour cela, cinq dimensions des dynamiques ont été déclinées en fonction de la qualité et de la quantité des changements induits, des mouvements spatiaux associés et des efFets sur le fonctionnement $\mathrm{du}$ territoire et sur sa trajectoire. Deux cas sont représentés systématiquement pour exemple mais c'est bien un large éventail de changements qui peut être modélisé.

Ce référentiel permet de modéliser les dynamiques qui opèrent la transition entre une situation présente et une situation future. Leur décomposition permet de mieux saisir la structure spatio-temporelle dans laquelle s'inscrit l'évolution des territoires.

La table des chorèmes spatio-temporels, initiée et éprouvée ici en prospective, pourrait également être généralisée à toute modélisation graphique spatio-temporelle. Elle contribue à penser la modélisation du temps en géographie et invite à la réflexion sur la production de modèles des processus spatiaux et des combinaisons spatiotemporelles-types.

\section{BIBLIOGRAPHIE}

ALLEN J.-F. (1983). « Maintaining knowledge about temporal intervals ». Communication of the ACM, vol. 26, n 11, nov. 1983, p. 832-843.

BAILly A., GLATRON M., MUSSO P. (2001). « Les images dans les travaux de prospective de la DATAR ». Territoires 2020, n 3, p. 11-25. En ligne : http://zonages.territoires.gouv.fr/sites/default/files/ datar/territoires2020-n-3-3.pdf

BIPE (2012). Territoires durables 2030 : un exercice de prospective. Rapport d'étude pour le Commissariat Général au Développement Durable, Délégation au développement durable, Mission Prospective du MEEDDM. Rapport d'étude, 100 p.

BONIN M. (2001). « Nouvelles fonctions de l'agriculture et dynamiques des exploitations. Une analyse chorématique dans les Monts d'Ardèche ». Mappemonde, $\mathrm{n}^{\circ} 62$ (2001/2), p. 11-16. En ligne : https://www.mgm.fr/PUB/Mappemonde/M201/Bonin.pdf

BRUNET R. (1986). « La carte-modèle et les chorèmes ». Mappemonde, (1986/4), p. 2-6. En ligne : https://www.mgm.fr/PUB/Mappemonde/M486/p2-6.pdf 
CASANOVA L. (2010). Les dynamiques du foncier à bâtir comme marqueurs du devenir des territoires de Provence intérieure, littorale et préalpine. Éléments de prospective spatiale pour l'action territoriale. Avignon : Université d'Avignon, thèse de doctorat de géographie, $441 \mathrm{p}$.

CASSINI Groupe TempsXEspace (1999). « Représentation de l'espace et du temps dans les SIG ». Revue internationale de géomatique. Hermès/Lavoisier, vol. 9 (1/1999), 124 p.

CGDD (2013). « Territoire durable 2030. Phase 1 : Enjeux et tendances ». Études \& Documents, $\mathrm{n}^{\circ} 93$.

CGDD (2012). « Territoire durable 2030. Une prospective de développement durable à l'échelle des territoires », Le point sur, $\mathrm{n}^{\circ} 124$.

CHEYLAN J.-P. (2007). « Les processus spatiaux-temporels : quelques notions et concepts préalables à leur représentation ». Mappemonde, $n^{\circ} 87$ (2007/3), 21 p. https://mappemonde-archive.mgm.fr/ num15/articles/art07303.html

CHEYLAN J.-P., DEFFONTAINES J.-P., LARDON S., THÉRY H. (1990). « Les chorèmes : un outil pour l'étude de l'activité agricole dans l'espace rural ? ». Mappemonde (4/1990), p 2-4. https://www.mgm.fr/ PUB/Mappemonde/M490/PREFACE.pdf

CHEYLAN J.-P., LIBOUREL T., MENDE C. (1997). « Graphical Modelling for Geographic Explanation ». Spatial Information Theory A Theoretical Basis for GIS. Proceedings, International Conference COSIT '97, Laurel Highlands, Pennsylvania, USA, October 15-18, p. 473-483.

DEBARBIEUX B. (2002). « Figures (géo-)graphiques et prospective. Cartes, schémas et modèles au service du projet et de la prospective territoriale ». In B. DEBARBIEUX et M. VANIER (dir.), Ces territorialités qui se dessinent, La Tour d'Aigues : Éditions de l'Aube/Paris : DATAR, coll. « Monde en cours. Série Bibliothèque des territoires ", 267 p. ISBN 2-87678-740-7.

DECOUPIGNY F. PASSEL F. (2014). « Réseaux de villes et détermination des pressions urbaines ». Cybergéo. https://journals.openedition.org/cybergeo/26410

DURAND-DASTÈs F. (1999). « Jamais deux fois, ou quelques précautions à prendre avec le temps ». Travaux de l'Institut de géographie de Reims, $\mathrm{n}^{\circ}$ 101-104, p. 5-23.

EPEES (2000). « Le concept d'évènement spatial ». L'Espace géographique, numéro spécial « L'évènement spatial en débat », n³, vol. 29, p. 200-217. https://www.persee.fr/doc/ spgeo_0046-2497_2000_num3_2006

GRATALOUP C. (1996). Lieux d'histoire. Essai de géohistoire systématique. Montpellier : Reclus ; Aubervilliers : La Documentation française, coll. « Espaces modes d'emploi », 200 p. ISBN 2-86912-065-6

HUREL K. (2010). « Toutes les cartes en main ». In DATAR (2010), « Aménager le changement ». Territoires 2040, $\mathrm{n}^{\circ}$ 1, Paris : La Documentation française, $103 \mathrm{p}$.

KADDOURI L. (2008). « Réflexion sur la sémiologie graphique animée des flux ». Mappemonde, $\mathrm{n}^{\circ} 89$, (2008/1), 12 p. https://mappemonde-archive.mgm.fr/num17/articles/art08104.html

KADDOURI L. (dir.), BLAISE J.-Y., DAVOINE P.-A., MATHIAN H., SAINT-MARC C. (2014). État des lieux des représentations dynamiques des temporalités des territoires. Rapport de recherche, PUCA, $167 \mathrm{p}$. http://webissimo.developpement-durable.gouv.fr/IMG/pdf/

Rapport_Annexes_PUCA_complet_final_cle587888.pdf

MABY J. (2002). Campagnes de recherche: Approches systémiques de l'espace rural. Avignon : Université d'Avignon, HDR, 2 vol. 
PÉGUY C.-P. (2001). Espace, temps, complexité : vers une métagéographie. Paris : Belin, coll.

«Géographiques », 283 p. ISBN 2-7011-2965-6

RODier X., GRATAloup C., GUilloteau C. (2010-2014). « Dossier : Chrono-chorématique urbaine ». Mappemonde, $\mathrm{n}^{\circ}$ 100, 105 et 114. https://mappemonde-archive.mgm.fr/dos_chrono.html

THÉRY H. (1990). « Chronochorèmes et paléochorèmes : la dimension temporelle dans la modélisation graphique ». In ANDRÉ Y., BAILLY A., CLARY M., FERRAS R., GUERIN J.-P., Modèles graphiques et représentations spatiales. Paris : Anthropos ; Montpellier : Reclus, coll. « Géographie », p. 41-61. ISBN 2-86912-031-5

UMR ESPACE, GAÏAGO, ECOVIA (2013). Géoprospective : modélisation et représentation graphique des scénarios de prospective pour un territoire durable à horizon 2030. Rapport de recherche, MEDDECommissariat général au développement durable - Mission Prospective, $124 \mathrm{p}$

\section{NOTES}

1. On oppose généralement les scénarios contrastés aux scénarios tendanciels car ils ont pour objectif de faire varier à l'extrême des hypothèses sur le futur.

2. Parmi les différents prestataires de services chargés de contribuer à l'élaboration des scénarios prospectifs les chercheurs de l'UMR Espace ont apporté un éclairage géoprospectif aux scénarios du programme de prospective nationale Territoire Durable 2030 pour le compte du Ministère de l'Environnement, du développement durable et de l'énergie.

3. Une démarche de prospective experte est réalisée par des spécialistes (scientifiques et/ou experts de la prospective) à partir de l'examen attentif d'études et/ou de séances de construction collective des hypothèses sur le futur.

4. Comme cela a été fait dans d'autres travaux de modélisation graphique (par exemple, Cheylan et al. 1990), le réseau est considéré comme une forme complexifiée de la ligne.

5. Extraits d'un raisonnement global exposé dans des vidéos disponibles http://www.territoiredurable-2030.developpement-durable.gouv.fr.

6. Développé par le BIPE (cabinet de conseil en analyse stratégique et prospective économique), le modèle DIVA-II est utilisé pour le chiffrage des évolutions macroéconomiques et sectorielles au niveau national et par région. Il s'agit d'un « modèle économétrique multisectoriel de long terme qui, à partir d'un ensemble cohérent d'hypothèses portant sur les principales variables macroéconomiques relatives à la France et au reste du monde, permet d'établir une projection des comptes "ressources-emplois" au niveau sectoriel en tenant compte de problématiques de demande, d'offre et de compétitivité au niveau international » (BIPE, 2012).

7. Les cartes sont consultables à l'adresse internet suivante: http://www.territoiredurable-2030.developpement-durable.gouv.fr/index.php/td2030/compare

8. Développée par Fabrice Decoupigny $(2001 ; 2014)$, FRED (FRéquentation Et Déplacement) est une plateforme de simulation utilisée pour le calcul d'indices de pression urbain de FRED. 


\section{RÉSUMÉS}

Cet article présente et éprouve à travers l'exemple de l'évolution des espaces urbains, une grille chorématique conçue pour modéliser les dynamiques spatiales (deux cas possibles sont déclinés pour exemple). Celles-ci opèrent la transition entre la situation de la France observée en 2010 et celles projetées en 2030, étudiées dans les trois scénarios Territoire Durable 2030 du Ministère de l'Écologie, du Développement Durable et de l'Energie (MEDDE). Les besoins de modélisation des dynamiques en prospective y sont discutés.

Using the evolution of urban spaces, this paper surveys and investigates a grid of "chorèmes" (elementary graphic structures) developed for the modelling of spatial dynamics (two case studies are presented). These dynamics span the transition from France's 2010 status to its projected 2030 status, as studied in the three scenarios presented by the Ministry of Ecology, Sustainable Development and Energy's program: Sustainable Territory 2030. The article discusses the needs for modelling prospective dynamics.

A través de dos ejemplos sobre la evolución de los espacios urbanos, este artículo prueba un conjunto de coremas que modelizan la dinámica espacial. Con ellos se visualiza la transición de Francia entre 2010 y sus proyecciones para 2030. Se realiza para los tres escenarios propuestos en el proyecto Territorio Sostenible 2030 del Ministerio de Ecología, Desarrollo Sostenible y Energía (MEDDE). Esto supone un debate metodológico sobre la necesidad de modelizar la dinámica prospectiva.

\section{INDEX}

Mots-clés : chorème, dynamique de changement, prospective, structure spatio-temporelle Palabras claves : corema, dinámicas de transformación, prospectiva, estructura espaciotemporal

Keywords : choreme, dynamic of change, prospective, spatio-temporal structure

\section{AUTEURS}

\section{LAURE CASANOVA ENAULT}

Université d'Avignon, UMR ESPACE 7300 CNRS

\section{CATHY CHATEL}

UNESP Brésil 